\title{
AVALIAÇÃO DA DIMENSÃO ESTRUTURA PARA A QUALIDADE DA ATENÇÃO PRIMÁRIA À SAÚDE*
}

Raissa Mont' Alverne Barreto ${ }^{1}$

Izabelle Mont' Alverne Napoleão Albuquerque ${ }^{2}$ Isabel Cristina Kowal Olm Cunha

Cibelly Aliny Siqueira Lima Freitas ${ }^{2}$

Jamilly Coelho Teixeira Braga ${ }^{1}$
https://orcid.org/0000-0001-7530-076X https://orcid.org/0000-0003-0856-5607 https://orcid.org/0000-0001-6374-5665 https://orcid.org/0000-0002-0585-5345 ttps://orcid.org/0000-0003-0291-9072

Objetivo: avaliar a qualidade da dimensão estrutura das Unidades Básicas de Saúde de Sobral, Ceará, a partir do referencial de Donabedian. Método: Trata-se de estudo avaliativo com abordagem quantitativa, realizada em novembro de 2018 a janeiro de 2019. Para coleta dos dados aplicou-se uma adaptação do Instrumento de Avaliação de Serviços de Atenção Básica com 18 gerentes e 46 equipes de saúde da família. Para análise dos dados foram utilizadas técnicas de estatística descritiva a partir da tabulação, processamento dos dados e produção de tabelas. Resultados: Observou-se ausência em 17\% das unidades de banheiro adaptado; ausência em 100\% das unidades de desfibrilador; 83\% delas referiram ausência de veículo para uso rotineiro de atividades pelos profissionais e constatou-se acesso regular de $78 \%$ para medicamentos de Hipertensão e apenas $61 \%$ para Diabetes. Conclusão: a estrutura das unidades precisa ser valorizada para qualificar as ações desenvolvidas na Atenção Primária à Saúde.

Descritores: Atenção à saúde; Avaliação em saúde; Atenção Primária à Saúde.

\section{EVALUATION OF THE STRUCTURE DIMENSION FOR THE QUALITY OF PRIMARY HEALTH CARE}

Objective: to evaluate the quality of the structure dimension of Basic Health Units in Sobral, Ceará, based on the Donabedian framework. Method: This is an evaluative study with a quantitative approach, carried out from November 2018 to January 2019. To collect the data, an adaptation of the Primary Care Services Evaluation Instrument was applied with 18 managers and 46 family health teams. For data analysis, descriptive statistics techniques were used based on tabulation, data processing and production of tables. Results: Absence was observed in $17 \%$ of the adapted bathroom units; absence of $100 \%$ of the defibrillator units; $83 \%$ of them reported the absence of a vehicle for routine use of activities by professionals and there was regular access of $78 \%$ for hypertension drugs and only $61 \%$ for diabetes. Conclusion: It is concluded that the structure of the units needs to be valued to qualify the actions developed in Primary Health Care.

Descriptors: Health care; Health evaluation; Primary Health Care.

\section{EVALUACIÓN DE LA DIMENSIÓN DE LA ESTRUCTURA PARA LA CALIDAD DE LAATENCIÓN PRIMARIA DE SALUD}

Objetivo: evaluar la calidad de la dimensión de la estructura de las Unidades Básicas de Salud en Sobral, Ceará, con base en el marco de Donabedian. Metodo: Este es un estudio evaluativo con un enfoque cuantitativo, llevado a cabo desde noviembre de 2018 hasta enero de 2019. Para recopilar los datos, se aplicó una adaptación del Instrumento de Evaluación de Servicios de Atención Primaria con 18 gerentes y 46 equipos de salud familiar. Para el análisis de datos, se utilizaron técnicas estadísticas descriptivas basadas en tabulación, procesamiento de datos y producción de tablas. Resultados: Se observó ausencia en el 17\% de las unidades de baño adaptadas; ausencia del 100\% de las unidades de desfibrilador; El 83\% de ellos reportó la ausencia de un vehículo para el uso rutinario de actividades por parte de profesionales y hubo un acceso regular del $78 \%$ para los medicamentos para la hipertensión y solo el 61\% para la diabetes. Conclusión: Se concluye que la estructura de las unidades necesita ser valorada para calificar las acciones desarrolladas en Atención Primaria de Salud.

Descriptores: Atención de salud; Evaluación de salud; Atención primaria de salud.

*Parte integrante da Dissertação de Mestrado apresentada à Universidade Federal do Ceará em 2019.

Universidade Federal do Ceará (UFC), Sobral, CE, Brasil.

${ }^{2}$ Universidade Estadual Vale do Acaraú (UVA), Sobral, CE, Brasil.

${ }^{3}$ Universidade Federal de São Paulo (UNIFESP), São Paulo, SP, Brasil.

Autor Correspondente: Raissa Mont'Alverne Barreto Email: raissinha_@hotmail.com Recebido: 05/3/20 Aceito: 12/5/20 


\section{INTRODUÇÃO}

É inegável o expressivo aumento de cobertura pela Estratégia Saúde da Família (ESF), o qual está diretamente implicado na melhoria dos indicadores de saúde da população. Porém, ainda há muitos problemas que precisam ser manejados para que a Atenção Primária à Saúde (APS) possa desempenhar seu papel de organizadora do sistema e coordenadora do cuidado, particularmente referidos à equidade do acesso, resolutividade e qualidade da atenção ${ }^{1,2}$.

Assim, autores ${ }^{1}$ acrescentam a carência de infraestrutura adequada nas unidades da ESF como problemas que ainda persistem nos dias de hoje. Nesse contexto, Donabedian ${ }^{3}$ defende que uma boa estrutura deve significar a probabilidade de um bom desempenho nas áreas de processo e de resultados.

Portanto, diante de tais desafios e tentativas constantes de estimular processos contínuos de melhoria de indicadores de acesso e qualidade na APS, entende-se que pesquisas avaliativas são necessárias para a construção de estratégias de intervenções mais adequadas que propiciem avanços no âmbito da produção de cuidados e gestão dos serviços de saúde ${ }^{4-6}$.

No Ceará, mais precisamente na cidade de Sobral, a ESF é baseada na singularidade e inovações do seu modelo tecnoassistencial de saúde, além de apresentar uma variedade de experiências exitosas dentro do contexto da $\mathrm{APS}^{7}$. Ao longo de quase 30 anos foram investidos muitos recursos na estruturação e manutenção dos Centros de Saúde da Família (CSF) do município, sendo importante, portanto, avaliar a qualidade da dimensão estrutura das Unidades Básicas de Saúde (UBS) nos dias de hoje, baseando-se no princípio de que a avaliação da qualidade deve ser uma atividade contínua para subsidiar o redirecionamento das práticas profissionais.

Neste sentido este estudo tem o objetivo de avaliar a qualidade da dimensão estrutura das Unidades Básicas de Saúde de Sobral, Ceará, a partir do referencial de Donabedian ${ }^{3}$.

Considera-se relevante este estudo de modo que as UBS estudadas possam identificar situações passiveis de mudanças e que possibilitem o alcance de metas favoráveis às necessidades reais da população assistida em cada UBS.

\section{MÉTODOS}

\section{Tipo de estudo}

Trata-se de uma pesquisa avaliativa, em que Hartz e $\mathrm{Silva}^{8}$ a define como o procedimento que consiste em fa- zer um julgamento de uma intervenção usando métodos científicos.

\section{Local do estudo}

O estudo foi realizado na APS da cidade de Sobral, Ceará entre os meses de novembro de 2018 a janeiro de 2019, contando com uma área de $2.122,897 \mathrm{~km}^{2}$ e uma população estimada, em 2019, de 208.935 habitantes'. A ESF do município possui ações e serviços de saúde configurados como objeto privilegiado de estudos, construção da integralidade como princípio em saúde e materializa o direito e o serviço na atenção e cuidado à saúde ${ }^{7}$.

\section{Participantes da pesquisa}

Participaram 18 gerentes das UBS pertencentes à zona urbana de Sobral, juntamente com os 46 demais profissionais de saúde que compreendem as equipes de saúde da família das respectivas Unidades, selecionados a partir de amostragem não-probabilistica por conveniência. Salienta-se que estes participantes representam os serviços de Atenção Básica que tiveram 100\% de adesão à pesquisa.

\section{Coleta de dados}

Foi realizada a aplicação de uma adaptação do Instrumento de Avaliação de Serviços de Atenção Básica (Qua$(i A B)^{10}$, visto que a metodologia original deste se destina a todos os serviços que compõem a rede de atenção básica, independentemente do modelo adotado, não sendo exclusiva para ESF. Nesta pesquisa, foram avaliados apenas os serviços pertencentes à ESF, respeitando-se as especificidades da realidade local, justificando, dessa forma, a necessidade de adaptação do referido instrumento.

Assim, esta adaptação consistiu em retirar questões do instrumento original que não estivessem ligadas diretamente à ESF para o alcance dos objetivos desta pesquisa, por exemplo: questões sobre o tipo de UBS (arranjos organizacionais) e gestão administrativa do serviço; e a inclusão de itens que contemplassem a especificidade local para conferir maior qualidade à pesquisa, mas sem distanciar-se da proposta original do questionário, como por exemplo, a inclusão de itens como sonar doppler, tensiomêtro, balança e termômetro na questão sobre equipamentos e recursos de apoio e inclusão dos medicamentos de hanseníase, tuberculose, anticoncepcionais orais e injetáveis, contracepção de emergência, ácido fólico, sulfato ferroso, verminoses, antitérmicos e analgésicos na questão sobre a dispensação de medicamentos.

Posteriormente, o instrumento adaptado para este estudo foi validado pela equipe de pesquisadores e profissionais do sistema municipal de saúde pertencentes ao Observatório de Pesquisas para o SUS (OBSERVASUS) de uma Universidade Estadual localizada no município es- 
tudado.

O QualiAB defende que todos os serviços de saúde na APS devem possuir um nivel aceitável de qualidade, de forma que o processo de construção de um novo modelo assistencial passa, necessariamente, pelo envolvimento ativo das equipes locais e por mudanças nas práticas de saúde ${ }^{11}$

A abordagem do QualiAB toma como referencial o de Donabedian com os indicadores de estrutura e processo, porém, para este estudo, utilizaram-se apenas os indicadores de estrutura das UBS de Sobral, os quais são referentes às características de espaço físico em termos de infraestrutura básica, equipamentos e insumos.

O questionário é composto por questões que os autores denominaram descritivas, as quais representam variáveis qualitativas, tais como localização, número de profissionais e suas respectivas formações, com vistas a caracterizar o serviço, atrelado às variáveis quantitativas de múltipla escolha, que são pontuadas em uma escala de 0,1 , e 2, sendo a pontuação 0 equivalente à ausência de recurso e/ou disponibilidade ou sua execução em patamares mínimos; a pontuação 1 correspondente à disponibilidade do recurso dentro do padrão médio esperado; e a pontuação 2 representando o melhor padrão possivel para o momento atual da rede básica.

Assim, a média aritmética da pontuação de todas as respostas atribui, ao serviço respondente, um grau de qualidade expresso pela distância do melhor padrão possível correspondente à média dois ${ }^{12}$.

Dessa forma, o instrumento foi desenhado para ser autorrespondido pelos serviços de Atenção Básica sob responsabilidade direta do gerente local, preferencialmente com a participação de toda a equipe. Assim, para aplicação online do questionário foi utilizado um software livre de plataforma web denominado LimeSurvey, e disponibilizado para cada UBS somente o link, via email, juntamente com seu token de acesso.

\section{Procedimento de análise dos dados}

Para suporte no processo de análise dos dados realizou-se a abordagem de estatística descritiva ${ }^{13}$ das respostas obtidas pelos serviços, organizadas em tabela e gráfico, em que as variáveis foram expressas por meio de porcentagem, e utilizou-se ainda a média aritmética da pontuação das questões e dos indicadores por UBS.

\section{Procedimentos éticos}

A pesquisa apresentou parecer favorável do Comitê de Ética em Pesquisas da Universidade Estadual Vale do Acaraú de acordo com a Resolução 466/2012 sob Parecer: 3.027.002, e os participantes assinaram o Termo de Con- sentimento Livre e Esclarecido.

\section{RESULTADOS}

Os resultados deste estudo foram apresentados e organizados em duas categorias: "Características Gerais dos serviços" e "Estrutura física, procedimentos e insumos básicos", as quais serão discorridas a seguir.

\section{Características Gerais dos serviços}

Das 22 UBS pertencentes à zona urbana do municipio de Sobral, 18 (82\%) participaram da pesquisa respondendo ao questionário. Destas, 10 (55,5\%) responderam o instrumento com a participação do gerente da unidade juntamente com a equipe de profissionais, destacando-se o profissional enfermeiro, e $8(44,5 \%)$ somente com a presença do gerente.

Quanto à distribuição das equipes de saúde da família por CSF em relação à quantidade de moradores e famílias, observou-se que nove CSF (50\%) não obedecem a quantidade ideal preconizada pela nova Política Nacional de Atenção Básica (PNAB), a qual recomenda a existência de uma equipe multiprofissional por 2000 a 3500 habitantes.

Verificou-se que dos 18 CSF estudados apenas 03 (17\%) estão com a equipe de saúde da família incompleta com a ausência do profissional médico. Além disso, dos 35 médicos integrantes das equipes dos $18 \mathrm{CSF}$, apenas quatro (11\%) são médicos de outras especialidades e 31 (89\%) são médicos de família e comunidade. fato este que fortalece a qualidade e longitudinalidade do cuidado em saúde pela capacidade do profissional de valorizar os atributos da APS.

O universo estudado apontou que há uma predominância do enfermeiro (83\%) ocupando a gerência das UBS, em que 12 deles (80\%) são do sexo feminino e três $(20 \%)$ do masculino. Os outros três gerentes (17\%) pertencem às categorias de Nutrição, Educação Física e Farmácia.

\section{Estrutura física, procedimentos e insumos básicos}

A tabela 1 mostra os indicadores selecionados. para esta dimensão avaliativa, segundo a média obtida por indicador, apontando os que alcançaram valores mais próximos e os que mais se distanciaram do padrão esperado representado pela pontuação máxima atribuída (média 2), sendo a porcentagem calculada a partir da média de cada indicador. Assim, observou-se que, entre dos cinco indicadores avaliados, nenhum obteve pontuação inferior a $50 \%$. 
Tabela 1 - Indicadores de qualidade quanto aos Recursos Materiais, Procedimentos e Insumos Básicos da APS do município de Sobral, CE, 2019.

\begin{tabular}{|l|c|c|}
\hline \multirow{2}{*}{ Indicador correspondente } & Média das UBS & Porcentagem (\%)Das UBS \\
\hline \multirow{2}{*}{ Insumos e Instalações da Sala de Vacina } & 2 & $100 \%$ \\
\cline { 2 - 3 } Procedimentos de rotina realizados na Unidade & 2 & $100 \%$ \\
\cline { 2 - 3 } Exames feitos ou colhidos na Unidade & 1,72 & $86 \%$ \\
\cline { 2 - 3 } Instalações Gerais & & \\
Equipamentos e Recursos de apoio disponiveis & 1,22 & \\
& & \\
\end{tabular}

De acordo com os dados coletados das 18 UBS, aqueles itens que não pontuaram por estarem ausentes nos CSF corresponderam, em maior quantidade, às salas e consultórios em número adequado para as atividades realizadas em 07 unidades (39\%), seguido de bom estado de conservação relacionado à pintura, móveis, instalações elétricas e hidráulica em 05 UBS (33\%); Ventilação e iluminações adequadas, corresponderam a 04 unidades (22\%); Sala para realização de atividades educativas e grupos a 03 UBS (17\%); Banheiro adaptado para pessoas portadoras de deficiência a 03 unidades (17\%); Sala de esterilização a 02 unidades (11\%) e Acesso à pessoas com deficiência a 01 unidade (6\%).

Para avaliação dos equipamentos e recursos de apoio disponiveis, obteve-se $61 \%$ do padrão esperado, em virtude da ausência de itens fundamentais para cobrir adequadamente as necessidades assistenciais, tais como desfibrilador (100\%); Oxímetro de pulso (94\%); Carrinho de emergência (83\%); Veículo para uso da Unidade (83\%); Cilindro de oxigênio (17\%); Acesso à ambulância para transporte de pacientes, quando necessário (11\%); Sonar doppler (11\%); Tensiômetro (11\%) e Acesso aos sistemas de informação para alimentação de banco de dados (6\%).

Outra questão apontada como ausência na maioria dos CSF deste estudo foi o veículo para uso da Unidade (83\%), o qual considera-se como parte dos recursos de suporte essenciais para atividades de rotina.

A disponibilidade ao conjunto de medicamentos previstos na RENAME (Relação Nacional de Medicamentos Essenciais) também foi um item avaliativo para contemplar a análise da estrutura da APS de Sobral, classificando-os em frequência regular; falta eventual; falta frequente e não dispensa esse grupo de medicamentos.

Observou-se que, para tuberculose e hanseníase, 94\% dos CSF referiram acesso regular para medicamentos relacionados a estas doenças. Por outro lado, a disponibilidade de medicamentos para hipertensão e diabetes foi classificada em $78 \%$ e $61 \%$ respectivamente para acesso regular nos CSF estudados, não obedecendo os limites desejáveis indicados pela Organização Mundial de Saúde (OMS).

A disponibilidade de Psicofármacos (utilizados na saúde mental) também foi priorizada como atributo de qualidade para esta dimensão avaliativa, tendo em vista o aumento de diagnósticos de transtornos psiquiátricos na população. Dessa forma, 66\% dos CSF estudados referiram acesso regular a este grupo de medicamentos, enquanto $28 \%$ apontou como falta eventual e $6 \%$ como falta frequente.

Os antibióticos, medicamentos para Infecções Sexualmente Transmissivel (IST) e para dislipidemias também fazem parte do grupo de medicamentos essenciais e constituem importante medida de acesso na ESF. Porém, os resultados deste estudo nos mostram que, do total de Unidades estudadas, $67 \%$ referiram acesso regular para o grupo de antibióticos, $56 \%$ para IST e $33 \%$ para dislipidemias.

\section{DISCUSSÃO}

O fato da pesquisa não ter alcançado $100 \%$ das respostas pode estar relacionado ao desafio de sensibilização e adaptação de gestores e profissionais ao processo de avaliação em saúde. Resultado como este também foi observado em uma pesquisa de autoavaliação da qualidade da ESF no âmbito da gestão de municípios de Minas Gerais, a 
qual revelou um baixo retorno dos questionários enviados aos participantes, não atingindo $100 \%$ de resposta para nenhum dos três instrumentos utilizados na pesquisa ${ }^{14}$.

Pesquisadores afirmam que, embora a avaliação em saúde seja um pressuposto da condição de gestão do sistema de saúde, essa ferramenta gerencial ainda não é utilizada em toda sua potencialidade. Porém, sabe-se da importância da participação destes profissionais nos processos avaliativos, visto que a avaliação favorece o desenvolvimento de processo crítico e reflexivo sobre as práticas desenvolvidas no âmbito dos sistemas de saúde ${ }^{12}$.

Importante salientar a presença significativa do enfermeiro no processo avaliativo da APS neste estudo. Porém, a coleta dos dados não possibilita uma análise mais qualificada sobre esse achado, mas nos revela que pode estar em desenvolvimento uma reconfiguração nos modos como esse profissional vem reorganizando seu processo de trabalho, afastando-se do modelo tradicional que tem dado suporte à formação e à própria historicidade da profissão, e aproximando-se da formulação, monitoramento e avaliação de políticas que incidem sobre os serviços de saúde ${ }^{13}$.

Em relação à presença significativa do enfermeiro na gerência das UBS, um estudo de revisão sobre as dimensões gerenciais na graduação em Enfermagem ${ }^{15}$ reforça este achado quando revela que o enfermeiro é reconhecido como o profissional que mais detém informações sobre o funcionamento dos serviços e, por isso, como uma de suas prerrogativas profissionais, o gerenciamento da equipe e dos serviços de Enfermagem têm aproximado o enfermeiro do papel de gerenciar unidades de saúde ${ }^{16}$.

Cabe ressaltar que as Diretrizes Nacionais Curriculares (DNC) para a Graduação em Enfermagem incluem os conteúdos de administração/gestão entre aqueles considerados imprescindiveis ao desenvolvimento das habilidades e competências profissionais e, talvez por este fato, os enfermeiros demonstram maior vocação, preparo e interesse para assumir as funções de gestor ${ }^{17}$.

Sobre os itens referentes à estrutura física das unidades, um estudo realizado em Caxias, interior do Maranhão, com o objetivo de avaliar a infraestrutura das UBS, encontrou resultados semelhantes ao deste estudo, em que revela que, dos itens analisados, a sala de vacina foi o quesito que obteve melhor avaliação, em contrapartida, a avaliação geral aponta que o município ainda peca em aspectos importantes, os quais não permitem oferecer ao público um serviço que contemple os padrões ideais em sua estrutura, como por exemplo, o número insuficiente para consultórios ${ }^{18}$.

De acordo com a nova PNAB, a ambiência de uma UBS é entendida como lugar social, profissional e de relações interpessoais, o qual deve proporcionar uma atenção acothedora para as pessoas, além de um ambiente saudável para o trabalho dos profissionais ${ }^{1}$. Assim, se faz necessário investir na estrutura física e ambiência das unidades, visto que para um ambiente adequado em uma UBS, existem componentes que atuam como modificadores e qualificadores do espaço.

Importante ressaltar a questão da adequação das Unidades para atender usuários portadores de deficiência, a qual também foi mencionada pela ausência em $17 \%$ das unidades de banheiro adaptado e em 11\% pela deficiência na acessibilidade, merecendo especial atenção, visto que tanto os serviços de saúde da família quanto os de média e alta complexidade devem garantir o amplo acesso e acessibilidade da comunidade, além de que, como qualquer outro grupo populacional, as pessoas com deficiência necessitam de acesso à saúde ${ }^{19}$

Estudos internacionais também enfatizam a necessidade de melhorias na acessibilidade, uma vez que a dificuldade de entrar em um estabelecimento é uma razão para pacientes com deficiência não procurarem os serviços de saúde. Assim, uma pesquisa desenvolvida na Índia com 839 pessoas com deficiência e 1153 sem deficiência revelou que aquelas com deficiência apresentaram uma necessidade de busca por serviços de saúde maior em comparação com as pessoas sem deficiência ${ }^{20,21}$.

A realidade do município de Sobral quanto à insuficiência de equipamentos adequados de urgência e emergência na ESF não se distancia dos demais no território brasileiro, em que uma pesquisa realizada em Campina Grande revelou inúmeros entraves para a adequada atenção às urgências na ESF, dentre eles, a falta de insumos e medicamentos mínimos de urgência foi um dos destaques que impedem os profissionais de proporcionar atendimento adequado $^{22}$. Porém, sabe-se que a ESF é responsável pelo acolhimento de todos os usuários e suas necessidades, inclusive das situações de urgências.

A nivel internacional também foram constatadas falhas no atendimento às urgências no nível primário de atenção. Estudos realizados em Alexandria, Egito e Arábia Saudita também detectaram falta de equipamentos e medicamentos essenciais para prestar assistência de qualidade aos seus usuários ${ }^{23,24}$

Nessa perspectiva, a Política Nacional de Atenção às Urgências (PNAU) preconiza que o atendimento de urgência seja realizado em qualquer nível de atenção à saúde, inclusive na APS, embora os profissionais desse nível de atenção não se reconheçam como responsáveis por esse 
tipo de assistência e, ao se depararem com situações de urgência, utilizem apenas o encaminhamento do paciente. Além disso, a PNAU recomenda que todas as unidades de saúde devem ter espaço físico adequado e materiais essenciais ao primeiro atendimento de urgência até que ocorra encaminhamento para os outros níveis de complexidade ${ }^{25}$.

O achado sobre a ausência de veículo em 83\% das unidades corrobora com um estudo realizado em Minas Gerais, o qual objetivou avaliar a infraestrutura e os equipamentos para ações na APS. Assim, a pesquisa também avaliou este item negativamente e considerou alarmante não haver um veículo para deslocamento para ações externas, o que compromete a disponibilidade para a assistência ao usuário e prejudica a qualidade do cuidado ${ }^{26}$.

Em relação à disponibilidade de medicamentos para hipertensão e diabetes, o resultado deste estudo foi semelhante ao de outro ${ }^{27}$, em que a prevalência de acesso a eles foi de $69 \%$ para hipertensos e $75 \%$ para diabéticos. Porém, de acordo com os parâmetros recomendados pela OMS, a qual preconiza indices superiores a $80 \%$, os percentuais apresentados são classificados como baixo a médio acesso, o que se pode inferir que ainda há hipertensos e diabéticos que necessitam comprá- $\operatorname{los}^{28}$.

Um estudo com representatividade nacional da amostra para as cinco regiões do Brasil e que obteve resultados similares ao deste, percebeu que as maiores prevalências de baixa adesão ao tratamento foram encontradas nos individuos que tiveram que pagar parte do seu tratamento em relação aos que tiveram o acesso gratuito a todos os medicamentos que necessitava para tratar as doenças crônicas referidas ${ }^{29}$. Este achado reforça que os medicamentos não fornecidos pelo SUS podem levar os usuários a não cumprir os tratamentos prescritos pela incapacidade de pagar ou de adquirir no setor privado ${ }^{30}$.

Um estudo com amostra nacional apresentou a menor disponibilidade aos psicotrópicos quando comparado aos outros medicamentos para o tratamento de Doenças Crônicas Não Transmissiveis, indicando um desafio à integralidade do cuidado, de forma que a possivel interrupção de tratamentos instituidos, decorrente do desabastecimento, pode ocasionar internações por doenças mentais e redução da qualidade de vida dos pacientes ${ }^{31}$.

Estes resultados refletem a necessidade de melhorar a disponibilidade destes medicamentos no nível primário de atenção para efetivar práticas integrais e resolutivas, e que torne possivel renovar as esperanças por um sistema de saúde digno, democrático e de qualidade para todos.

Considerando o cenário estudado e as realidades se- melhantes no território nacional observadas por meio da discussão na literatura científica, verifica-se a necessidade de maior compromisso político com a prioridade da ESF, de forma a obter melhorias para ampliar e qualificar os insumos básicos, infraestrutura, equipamentos clínicos e de apoio. Porém, o que se vivencia na atual conjuntura política e econômica do País é um grave contexto de redução do financiamento do SUS, considerado um grande retrocesso que restringe sua atuação.

\section{Limitações do estudo}

Destaca-se o fato de terem sido avaliadas apenas as UBS da zona urbana de Sobral, podendo ser estendido esse processo avaliativo para a zona rural. Também podem ser citadas as limitações inerentes à pesquisa avaliativa sujeita às limitações próprias desse tipo de delineamento.

\section{Contribuições para a prática}

Os resultados permitem a obtenção de informações essenciais à tomada de decisões estratégicas pelos gestores, e contribuem para a elaboração de um planejamento baseado nas necessidades e proposições de melhorias para a qualificação da assistência prestada à população do municipio estudado.

\section{CONCLUSÃO}

Os resultados evidenciaram condições básicas para o desenvolvimento de ações de qualidade para a prestação do cuidado. Porém, vale ressaltar a identificação de aspectos que podem comprometer esta assistência, como a necessidade de melhorias na acessibilidade para portadores de deficiência, visto que observou-se ausência em 17\% das unidades de banheiro adaptado e em $11 \%$ houve deficiência na acessibilidade; Insuficiência de equipamentos adequados de urgência e emergência, em que verificou-se ausência em $100 \%$ das unidades de desfibrilador e em $94 \%$ de oxímetro de pulso.

Além disso, 83\% das unidades referiram ausência de veículo para uso rotineiro de atividades pelos profissionais e constatou-se que houve acesso regular de $78 \%$ para medicamentos de Hipertensão e apenas 61\% para Diabetes.

Ressalta-se que as inadequações dos CSF prejudicam o desenvolvimento das ações na APS e geram insatisfação nos profissionais e usuários. Assim, é importante que a estrutura seja discutida e valorizada na avaliação dos serviços de saúde, no sentido de aumentar o impacto e qualificar as ações desenvolvidas na APS.

Contribuição dos Autores: RMAB, IMANA, ICKOC, CASLFC, JCTB, RBS: Concepção e desenho, análise e interpretação dos dados, redação do artigo, revisão crítica, revisão final. 


\section{REFERÊNCIAS}

1.Facchini LA, Tomasi E, Dilélio AS. Qualidade da Atenção Primária à Saúde no Brasil: avanços, desafios e perspectivas. Saúde debate[internet]. 2018 [acesso 2019 jun 14]; 42 (esp l):208-223. Disponivel em: http://www. scielo.br/pdf/sdeb/v42nspel/0103-1104-sdeb-42-spe01-0208.pdf.

2.Feitosa RMM, Paulino AA, Lima Junior JOS, Oliveira KKD, Freitas RJM, Silva WS. Mudanças ofertadas pelo Programa Nacional de Melhoria do Acesso e da Qualidade da Atenção Básica. Saúde Soc. [internet]. 2016 [acesso 2019 jun 10]; 25(3):821-829. Disponível em: http://www.scielo.br/pdf/sausoc/v25n3/1984-0470sausoc-25-03-00821.pdf.

3.Donabedian A. The definition of quality and approaches to its assessment. In: Donabedian A. The quality of medical care: a concept in search of a dOefinition. J Fam Pract. [internet]. 1979 agosto [acesso 2018 dez 18]; 9(2):277-84. Disponivel em: https://www.ncbi.nlm.nih.gov/pubmed/110905.

4.Araújo AC, Freitas FRNN, Araújo RV, Batista MMSL. Qualidade dos serviços na estratégia saúde da família sob interferência doPMAQ-AB: análisereflexiva. RevenfermUFPEonline[internet].2017[acesso2020maio 27];11(2):7127. Disponivel em: https://webcache.googleusercontent.com/search?q=cache:rBgysopbPv8J:https://periodicos. ufpe.br/revistas/revistaenfermagem/article/download/11990/14554+\&cd=18hl=pt-BREct=clnkधgl=br

5. Pinto Junior EP, Cavalcante JLM, Sousa RA, Morais APP, Silva MGC. Análise da produção científica sobre avaliação, no contexto da saúde da família, em periódicos brasileiros. Saúde debate [internet]. 2015 [acesso 2020 maio 27]; 39(104): 268-278. Disponivel em: https://www.scielo.br/pdf/sdeb/v39n104/0103-1104-sdeb-39-104-00268.pdf. 6.Silva CSO, Fonseca ADG, Souza LPS, Siqueira LG, Belasco AGS, Barbosa DA. Integralidade e Atenção Primária à Saúde: avaliação sob a ótica dos usuários. Ciênc. Saúde Coletiva [internet]. 2014 [acesso 2020 maio 27];19(11):44074415. Disponivel em: https://www.scielo.br/pdf/csc/v19nll/1413-8123-csc-19-11-4407.pdf.

7. Linhares JH, Frota SLF, Soares CHA, Linhares AEMS, Melo MSS. Sistema de informação em fisioterapia na atenção básica no território dos Terrenos Novos no municipio de Sobral, Ceará. SANARE [internet]. 2010 [acesso 2019 jan 17]; 9(1): 14-16. Disponivel em: https://sanare.emnuvens.com.br/sanare/article/view/198/185.

8. Hartz ZMA, Silva LMV da. Avaliação em saúde: dos modelos teóricos à prática na avaliação de programas e sistemas de saúde [internet]. Salvador: EDUFBA; Rio de Janeiro: Fiocruz, 2005 [acesso 2018 nov 12 ]. Disponivel em: http://books.scielo.org/id/xzdnf/pdf/hartz-9788575415160.pdf

9. Instituto Brasileiro de Geografia e Estatistica (IBGE). Cidades, Ceará, Sobral [internet]. 2019 [acesso 2020 maio 29]. Disponivel em: https://cidades.ibge.gov.br/brasil/ce/sobral/panorama.

10. Caderno de boas práticas para organização dos serviços de atenção básica: Critérios e padrões de avaliação utilizados pelo Sistema QualiAB. Organização e autoria Castanheira ERL, et al. Botucatu: UNESP-FM [internet]. 2016 [acesso 2020 maio 29]. Disponivel em: http://www.abasica.fmb.unesp.br/doc/CADERNO.pdf

11.Castanheira ERL Nemes MIB, Zarilli TFT, Sanine PR, Corrente JE. Avaliação de serviços de Atenção Básica em municipios de pequeno e médio porte no estado de São Paulo: resultados da primeira aplicação do instrumento QualiAB. Saúde debate [internet]. 2014 out-dez [acesso 2018 out 25]; 38(103): 679-691. Disponível em: http://www. scielo.br/pdf/sdeb/v38n103/0103-1104-sdeb-38-103-0679.pdf.

12. Carvalho Filha FSS, Nogueira LT, Medina MG. Avaliação do controle de hipertensão e diabetes na Atenção Básica: perspectiva de profissionais e usuários. Saúde debate [internet]. 2014 [acesso 2019 mar 16]; 38(esp.): 265278. Disponivel em: http://www.scielo.br/pdf/sdeb/v38nspe/0103-1104-sdeb-38-spe-0265.pdf.

13. Galavote HS, Zandonadel E, Garcia ACP, Freitas PSS, Seild H, Contarato PC, et al. O trabalho do enfermeiro na Atenção Primária à Saúde. Esc Anna Nery [internet]. 2016 [acesso 2019 mar 16]; 20(1): 90-98.Disponivel em: http:// www.scielo.br/pdf/ean/v20nl/1414-8145-ean-20-01-0090.pdf.

14.Cardoso AVL, Chain APN, Mendes RIP, Ferreira EF, Vargas AMD, Martins AMEBL, et al. Avaliação da gestão da estratégia saúde da família por meio do instrumento avaliação para melhoria da qualidade em municipios de Minas Gerais, Brasil. Ciên. Saúde Coletiva [internet]. 2015 [acesso 2019 jan 10]; 20(4): 1267-1284. Disponivel em: http://www.scielo.br/pdf/csc/v20n4/pt_1413-8123-csc-20-04-01267.pdf

15. Barreto RMA, Vasconcelos MN, Melo ES, Araújo MAF, Lira RCM, Albuquerque IMN. Dimensões gerenciais na formação acadêmica de enfermagem: uma revisão integrativa. Rev. Eletr. Enf [internet]. 2018 [acesso 2019 mar 26]; 20: 20-27. Disponível em: https://www.revistas.ufg.br/fen/article/view/47945/26644.

16. Mattos JCO, Balsanelli AP. A liderança do enfermeiro na atenção primária à saúde: revisão integrativa. Rev. 
Enferm. Foco. 2019; 10 (4): 164-171. Disponivel em: http://revista.cofen.gov.br/index.php/enfermagem/article/ view/2618/621.

17.Ministério da Educação (BR). Conselho Nacional de Educação, Câmara De Educação Superior. Resolução CNE/CES № 3, de 7 de novembro de 2001. Institui Diretrizes Curriculares Nacionais do Curso de Graduação em Enfermagem. Diário Oficial da União. 08 Nov 2001.

18. Gomes RNS, Portela NLC, Pedrosa AO, Monte LRS, Cunha JDS, Soares TR. Avaliação da estrutura física de Unidades Básicas de Saúde. Rev Rene [internet]. 2015 [acesso 2018 set 14]; 16(15): 624-30. Disponivel em: http://www.periodicos.ufc.br/rene/article/view/2777/2157.

19. Martins KP, Costa TF da, Medeiros TM de, et al. Internal structure of Family Health Units: access for people with disabilities. Ciênc. Saúde coletiva [internet]. 2016 out[acesso 2019 mar 23]; 21(10): 3153-3160. Disponivel em: http://www.scielo.br/pdf/csc/v2ln10/1413-8123-csc-21-10-3153.pdf.

20. Gudvalleti MVS, John N, Allagh K, Sagar J, Kumar S, Ramachandra SS. Access to health care and employment status of people with disabilities in South India, the SIDE (South India Disability Evidence) study. BMC Public Health [internet]. 2014 nov [acesso 2019 fev 22]; 14(1): 1125. Disponivel em: https://www.ncbi.nlm. nih.gov/pubmed/25361570.

21. Lagu T, Iezzoni LI, Lindenauer PK. The axes of access - improving care for patients with disabilities. N Engl J Med [internet]. 2014 maio [acesso 2019 fev 22]; 370 (19). Disponivel em: https://www.aahd.us/wp-content/ uploads/2012/10/AxesofAccess-ImprovingCareforPatientswithDisabilities-NEJM2014.pdf.

22.Farias DC, Celino SDM, Peixoto JBS, Barbosa ML, Costa GMC. Acolhimento e Resolubilidade das Urgências na Estratégia Saúde da Familia. Revista Brasileira de Educação Médica [internet]. 2015 [acesso 2019 mar 02]; 39(1): 79-87. Disponivel em: http://www.scielo.br/pdf/rbem/v39n1/1981-5271-rbem-39-1-0079.pdf.

23. Mohey A, Al Azmi SF. Primary Healthcare Emergency Services in Alexandria, Egypt 2016. Quality in Primary Care [internet]. 2017 [ acesso 2019 abril 10]; 25 (5):303-315. Disponivel em: http://primarycare.imedpub.com/ primary-healthcare-emergency-services-in-alexandria-egypt-2016.php?aid=20772

24.Alsaad SSM, Abu-Grain SHS, El-Kheir DYM. Preparadness of Dammam Primary Health Care centers to deal with emergency cases. Jounal of Family and Community Medicine [internet]. 2017 set/dez [acesso 2019 abril 21]; 24 (3): 181-188. Disponivel em: https://www.ncbi.nlm.nih.gov/pubmed/28932163.

25. Ministério da Saúde (BR). Política Nacional de Atenção às Urgências. 3.ed. Brasília: Ministério da Saúde; 2006.

26. Moreira KS, Lima CA, Vieira MA, Costa SM. Avaliação da infraestrutura das unidades de saúde da familia e equipamentos para ações na Atenção Básica. Cogitare Enferm [internet]. 2017 [acesso 2019 fev 04]; 22(2): e51283. Disponível em: https://revistas.ufpr.br/cogitare/article/view/51283/pdf.

27. Barreto MNSC, Cesse EAP, Lima RF, Marinho MGS, Specht YS, Carvalho EMF, et al. Análise do acesso ao tratamento medicamentoso para hipertensão e diabetes na Estratégia de Saúde da Família no Estado de Pernambuco, Brasil. Rev. bras. Epidemiol [internet]. 2015 abril/junho [acesso 2019 fev 10]: 18(2): 413-424. Disponivel em: http://www.scielo.br/pdf/rbepid/v18n2/pt_1415-790X-rbepid-18-02-00413.pdf.

28. World Health Organization (WHO). Access to essential medicines. In: WHO. The World Medicine Situation. Geneva: WHO; 2004. p. 61-74.

29.Tavares NUL, Bertoldi AD, Mengue SS, Arrais PSD, Luiza VL, Oliveira MA, et al. Fatores associados à baixa adesão ao tratamento farmacológico de doenças crônicas no Brasil. Rev. Saúde Pública [internet]. 2016 [acesso 2018 dez 15]; 50 (supl. 2): 10s. Disponivel em: http://www.scielo.br/pdf/rsp/v50s2/pt_0034-8910-rsps2-S01518-87872016050006150.pdf.

30.Santos ATF, Silva ET, Larré MC, Inagaki ADM, Silva JRS, Abud ACF. Fatores associados à baixa adesão ao tratamento farmacológico de doenças crônicas no Brasil. Rev. Enferm. Foco 2019; 10 (1): 65-70 Disponível em: http://revista.cofen.gov.br/index.php/enfermagem/article/view/1348/497.

31. Nascimento RCRM, Álvares J, Guerra Junior AA, Gomes IC, Costa EA, Leite SN, et al. Disponibilidade de medicamentos essenciais na atenção primária do Sistema Único de Saúde. Rev Saude Publica [internet]. 2017 [acesso 2019 fev 04]; 51 (supl. 2): 10s. Disponivel em: http://www.scielo.br/pdf/rsp/v51s2/pt_0034-8910-rspS1518-51-s287872017051007062.pdf. 